\title{
Relationship between Diet, Cosmetics and Degree of Acne Vulgaris in Dermatovenereology Outpatients at Dr. Soetomo General Hospital, Surabaya
}

\author{
Mah Zhook Yueng, ${ }^{1}$ Diah Mira Indramaya, ${ }^{2}$ Arifa Mustika $^{3}$ \\ ${ }^{1}$ Faculty of Medicine Universitas Airlangga Surabaya, Indonesia, ${ }^{2}$ Department of Dermatology \\ and Venereology Faculty of Medicine Universitas Airlangga/Dr. Soetomo General Hospital \\ Surabaya, Indonesia, ${ }^{3}$ Department of Pharmacology Faculty of Medicine Universitas Airlangga \\ Surabaya, Indonesia
}

\section{Abstract}

Background: Acne vulgaris (AV) is a chronic inflammatory multifactorial disease that involves the pilosebaceous unit. The lesions of acne may be either non-inflammatory (comedones, which are blackheads or whiteheads) or inflammatory lesions (papules, nodules or pustules). The effects of diet and cosmetics on acne are still inconclusive. The objective of this study was to analyse the relationship between diet, cosmetics and the degree of AV.

Methods: This was a hospital-based, analytic observational study with cross-sectional design. The subjects were patients from the Outpatient Clinic of Dermatovenereology Department of Dr. Soetomo General Hospital. The questionnaire was filled by the respondents and the degree of AV was graded by the specialist.

Results: Among the types of diet, white bread $(p=0.039)$, chocolate $(p=0.044)$ and oily food $(p=0.013)$ are significantly associated with the degree of AV. However, white rice, potatoes and milk were insignificantly related to degree of $\mathrm{AV}(\mathrm{p}>0.05)$. No significant association was found in cosmetics (cleanser, moisturizer, facial protection, exfoliators and decorative cosmetics) with the degree of $\mathrm{AV}$ $(\mathrm{p}>0.05)$.

Conclusions: The factors that have an influence on the degree of AV are white bread, chocolate and oily food intake.

Keywords: Acne vulgaris, cosmetics, degree of acne, diet, multifactorial disease

\section{Introduction}

Acne vulgaris (AV) is a chronic obstructive and inflammatory dermatoses, seen mainly in adolescent and involves the sebaceous follicle. Scarring and pigmentation might be caused by the characteristic lesions such as open (black) and closed (white) comedones, inflammatory papules, pustules, nodules and cysts. ${ }^{1}$ Acne vulgaris is a condition which is caused by multiple factors.

However, the pathogenic factors involved are revolved around the interaction between follicular hyperkeratinisation, colonization of $P$. acnes, increased sebum production and inflammation. The factors that will cause acne vulgaris includes, hormonal, genetics, diet, stress, cosmetics etc. ${ }^{1}$

Lately, dietary factors such as insulin resistance and dietary carbohydrates have been associated with the etiology of acne. Some people believe chocolate and oily foods are the culprit of getting acne. Milk is also commonly associated with the occurrence of acne. Many recent studies investigate the role of diet as the cause of acne vulgaris. A few studies were carried out to support the hypothesis that high glycemic index (GI) diet and dairy products are related to this skin condition. However, the effects of diet on acne are still controversial. Other than diet, cosmetics which is widely used especially among generation $\mathrm{Y}$ and $\mathrm{Z}$ is also one of the factors that will influence the occurrence of acne vulgaris. Some cosmetic products that increase the risk of whiteheads

Correspondence: Mah Zhook Yueng, Faculty of Medicine Universitas Airlangga, Jl. Professor Dr. Mustopo No.47, Pacar Kembang, Jawa Timur, Indonesia, Email: zhookyueng_1995@hotmail.com 
and blackheads occurrences are known as comedogenic. When the inducing ingredient gets into the hair follicles and pores, it will clog them up and leads to sebaceous gland blockage due to the excess oil within the content of the product. ${ }^{2}$ Yet, usage of oil-free concealers, noncomedogenic makeup can enhance the quality of life of individuals and does not make the condition worse. ${ }^{3}$ Removing excessive oil or dirt from the face can be done by cleansing the face. Nevertheless, washing frequently to remove excessive oil is ineffective as it aggravates the sebaceous gland and causes oiliness to rebound. Moreover, using exfoliators and scrubbing can also irritate the sebaceous gland and leads to trauma which worsens the condition of acne. ${ }^{4}$

Information on diet and cosmetics influence the degree of acne vulgaris which is important for the management of the skin condition and education for the community to prevent and recover from the acne condition. Hence, this study was aimed to analyse the relationship between diet, cosmetics and the degree of acne vulgaris.

\section{Methods}

This study was an analytic observational, cross-sectional study carried out at the Dermatovenereology Outpatient Clinic of Dr. Soetomo General Hospital, Surabaya during March-July 2018. The ethical clearance of this study was issued by the Health Research Ethics Committee of Dr. Soetomo General Hospital, Surabaya. Respondents were selected using the random sampling method and 42 respondents were included in this study.

The respondents were interviewed using a structured questionnaire after obtaining their informed consent. The types of food investigated were white bread, white rice, potatoes, chocolate, oily food and milk. The frequency of food consumption was recorded

\section{Table 1 Characteristics of Respondents}

\begin{tabular}{lcc}
\hline \multicolumn{1}{c}{ Characteristics of respondents } & No. of respondents & \% \\
\hline Age (years) & 6 & 14.3 \\
$\leq 16$ (early adolescent) & 31 & 73.8 \\
17-25 (late adolescent) & 3 & 7.1 \\
$26-35$ (early adult) & 2 & 4.8 \\
36-45 (late adult) & & \\
Sex & 38 & 90.5 \\
Female & 4 & 9.5 \\
Male & & \\
Marital status & 36 & 85.7 \\
$\quad$ Not married & 6 & 14.3 \\
Married & & \\
Educational status & 6 & 14.3 \\
Junior High School & 17 & 40.5 \\
Senior High School & 19 & 45.2 \\
University & & \\
Occupation & 27 & 64.3 \\
Student & 3 & 7.1 \\
Housewife & 8 & 19.0 \\
Private sector & 4 & 9.5 \\
Others & 42 & 100 \\
Total & &
\end{tabular}


Mah Zhook Yueng, Diah Mira Indramaya, Arifa Mustika: Relationship between Diet, Cosmetics and Degree of 163 Acne Vulgaris in Dermatovenereology Outpatients at Dr. Soetomo General Hospital, Surabaya

Table 2 Distribution of Degree of Acne Vulgaris

\begin{tabular}{|c|c|c|}
\hline Degree of AV & No. of respondents & $\%$ \\
\hline Mild & 27 & 64.3 \\
\hline Moderate & 11 & 26.2 \\
\hline Severe & 4 & 9.5 \\
\hline Total & 42 & 100 \\
\hline
\end{tabular}

after being divided into five categories, namely 'none', 'less than 4 times/month', '1 time/ week', '2-4 times/week' and '5-7 times/week'. Cosmetics were categorized as cleansers, moisturizers, facial protection, exfoliators and decorative cosmetics. Usage of all five types of cosmetics were recorded in this study, respondents answered 'yes' if they use the products and answered 'no' if they do not use it. Frequency of face wash was recorded after being grouped into 'none', '1-2 times/day' and 'more than 3 times/day'. Frequency of applying decorative cosmetics was recorded after being classified into 5 groups, which is 'none', 'only on special occasions', ' 1 time/week', '2-4 times/week' and '5-7 times/week'. The type of cleansers, moisturizers, and decorative cosmetics were also recorded in this study.

Part One and Part Two of Baumann Skin Type Indicator had been translated into the Indonesian language and were included in the questionnaire to study the relation of skin type and skin sensitivity with degree of acne. Other factors such as hormonal, genetics, stress, infection and trauma were also included in the questionnaire. The established face validity method is used to validate the questionnaire.

The diagnosis of the degree of acne was carried out by the specialist of the Dermatovenereology Outpatient Clinic at Dr.

Table 3 Relationship between Diet and Degree of Acne Vulgaris

\begin{tabular}{lc}
\hline \multicolumn{1}{c}{ Diet } & p-value \\
\hline White bread & $0.039^{*}$ \\
White rice & 0.293 \\
Potatoes & 0.511 \\
Chocolate & $0.044^{*}$ \\
Oily food & $0.013^{*}$ \\
Milk & 0.416 \\
\hline Note. ${ }^{*}$ significantly significant $\mathrm{p}<0.05$ &
\end{tabular}

Soetomo General Hospital, Surabaya using the Lehmann grading system. The number of comedones, inflammatory lesions and total lesion counts were noted to classify the degree of acne into mild, moderate and severe. Data analysis was performed using the chi-square test with the Statistical Package for Social Sciences (SPSS), Version 21.

\section{Results}

Among 42 respondents, 38 respondents $(90.5 \%)$ were females and 4 respondents $(9.5 \%)$ were males. The majority of the respondents $(73.8 \%)$ were between 17 25 years old with a mean age of $21.90 \pm 6.5$ years old. The characteristics of most of the respondents were unmarried $(85.7 \%)$; with a university degree (45.2\%); currently still a student (64.3\%).

Acne patients were divided into 3 groups according to the number of comedones, inflammatory and total lesion count by using the Lehmann grading system. It was found that the majority of the respondents hadmild acne (64.3\%).

Based on the study on dietary factors, only white bread, chocolate and oily food influenced the degree of acne vulgaris (Table 1). Using the Chi-square Test, the degree of acne was not influenced by cosmetics products (Table 4). Of the seven other acne aggravators included in the questionnaire, none of them influenced the degree of acne (Table 5).

\section{Discussion}

Among the different diets studied in this research, white bread, chocolate, and oily food had a significant positive relationship with the degree of acne vulgaris whereas white rice, potatoes and milk did not have any significant relation with the degree of acne vulgaris.

According to Atkinson et al. ${ }^{5}$, white bread, white rice and potatoes are foods with high GI. Among the foods with high GI studied in this 
Table 4 Relationship between Cosmetics and Degree of Acne Vulgaris

\begin{tabular}{lc}
\hline \multicolumn{1}{c}{ Cosmetics } & p-value \\
\hline Cleansers & \\
Usage of cleansers & 0.287 \\
Frequency of face wash & 0.744 \\
Types of cleansers & \\
$\quad$ Cleansing cream & 1.000 \\
$\quad$ Cleansing milk & 0.686 \\
Face tonic & 1.000 \\
$\quad$ Skin freshener & 0.739 \\
Facial mask & 0.330 \\
Moisturizers & \\
Usage of moisturizers & 0.242 \\
Types of moisturizers & \\
$\quad$ Morning cream & 1.000 \\
$\quad$ Night cream & 1.000 \\
$\quad$ Moisturizing cream & 0.639 \\
Facial Protection & \\
Usage of facial protection (Sunblock) & 1.000 \\
Exfoliators & \\
Usage of exfoliators & 0.686 \\
Decorative cosmetics & \\
Usage of decorative cosmetics & \\
Frequency of applying decorative cosmetics & 1.000 \\
Types of decorative cosmetics & 0.761 \\
$\quad$ Make up base & \\
$\quad$ Make up foundation & 0.273 \\
$\quad$ Primer & 0.750 \\
Blusher & 0.451 \\
\hline & 1.000 \\
\hline
\end{tabular}

study, only white bread was associated with the degree of acne vulgaris whereas white rice and potatoes had no relation with the degree of acne vulgaris. In contrast to this study, Di Landro et al. ${ }^{6}$ states that the intake of bread has no relation with the risk of acne (OR 1.39, 95\% CI 0.65-2.98). A study in New York ${ }^{7}$, compared the dietary GI among participants with no acne, mild acne and moderate to severe acne. The study shows that the group with moderate to severe acne (51.8 \pm 3.0$)$ have a higher dietary GI than the group with no acne $(48.9 \pm 4.6)$ and mild acne (49.6 \pm 3.9 ). The study also suggests that dietary GI could affect or aggravate acne development $(\mathrm{p}<0.001)$. $^{7}$
Dietary factors particularly high glycemic load diets are also proven to have an association with the development of $\mathrm{AV}$, as shown in a casecontrol study conducted in Malaysia ${ }^{8}$. Patients with $\mathrm{AV}$ have a significantly higher dietary glycemic load $(175 \pm 35)$ compared to controls $(122 \pm 28)(p<0.001) .{ }^{8}$ The GI of meals and insulin response have a direct correlation; high GI diets have been shown to increase insulin resistance. The increased proliferation of basal keratinocytes due to high insulin concentration in the fasting and/or post prandial state may exacerbate acne. The synthesis of androgens is also stimulated by insulin, which leads to high sebum production, a recognized correlate 
Mah Zhook Yueng, Diah Mira Indramaya, Arifa Mustika: Relationship between Diet, Cosmetics and Degree of 165 Acne Vulgaris in Dermatovenereology Outpatients at Dr. Soetomo General Hospital, Surabaya

Table 5 Relationship between Other Factors and Degree of Acne Vulgaris

\begin{tabular}{lc}
\hline \multicolumn{1}{c}{ Factors } & p-value \\
\hline Skin Type & 0.638 \\
Skin Sensitivity & 0.689 \\
Hormonal & 1.000 \\
$\quad$ Male & 0.689 \\
$\quad$ Female & 0.734 \\
Genetics & 0.730 \\
Stress & \\
Infection and Trauma & 0.089 \\
$\quad$ Habit of scraping, scratching, picking or digging the skin & 0.287 \\
Habit of shaving, waxing, threading or performing laser hair removal & \\
\hline
\end{tabular}

of acne severity. Inflammatory responses enclosed and adjacent to the comedone could be increase due to insulin resistance. The significant relation between chocolate and oily food provides further support to a cross-sectional study which shows that sweet and oily food are an aggravating factor for moderate to severe acne ( $\mathrm{P}=0.03, \mathrm{X} 2$ test $){ }^{9}{ }^{9}$

In another prospective, a placebo-controlled study states that there is a significant increase of total acneiform lesions (comedones, papules, pustules, nodules) and noninflammatory lesion on day $4(\mathrm{p}=0.006, \mathrm{p}=0.009$ respectively) and day $7(p=0.043, p=0.042$ respectively) after consuming chocolate. ${ }^{11}$ Both studies showed that consumption of chocolate correlates to an increase in the exacerbation of acne. However, in contrast with the high prevalence of belief, Adebamowo et al. ${ }^{12}$ conducted a study with more than 4000 boys, the study shows no associations between acne and intakes of chocolate and French fries. Currently, there are not many studies that conclude chocolate or any specific fatty foods will cause acne, but it is clear that sebum production will increase as we consumed high-sugar/high-fat diet. Thus, promote inflammatory responses in the body. ${ }^{10}$

This study is in line with a study by Cerman et al. ${ }^{13}$ which shows no significant difference between milk and acne vulgaris subgroups (mild acne, moderate acne and severe acne) $(p=0.596, p>0.05)$. Besides, the study also shows no significant association between dairy product consumption among acne vulgaris and control groups ( $\mathrm{p}=0.911, \mathrm{p}>0.05)$. Yet, the data of this study only covers the milk consumption in the previous 7 days of the study, this result may not reflect the long-term consumption habits. ${ }^{13}$ In contrast with this study and the above study, a case-control study by Di Landro et al. ${ }^{6}$ states that a diet with higher milk consumption influenced the risk of moderate to severe acne regardless of the family history and BMI. Skim milk (OR 2.20, 95\% CI 1.184.10) has a more prominent association with the risk of moderate to severe acne than whole milk (OR 1.64, 95\% CI 0.81-3.33) although the difference does not reach any statistical significance. ${ }^{7}$ A study performed by Adebamowo et al. ${ }^{12}$ which includes more than 4000 boys also shows a positive correlation between consumption of skim milk and acne vulgaris $(p=0.02, p<0.05)$. Thus far, there has been no conclusive evidence that milk and dairy products have comedogenic effects.

Cosmetics are categorized into cleanser, moisturizer, facial protection, exfoliators and decorative cosmetics. There was no significant relation between frequency of face wash and degree of acne vulgaris in this study. It is believed that exacerbation of the condition occurs when excessive washing of acne afflicted area occurs. ${ }^{4}$ A study conducted by Rahmawati et al. ${ }^{14}$ in Semarang, Indonesia, reports that there is no relation between frequency of face wash with the occurrence of mild-moderate and severe acne. The study also suggested the ideal face wash frequency is insufficient; the correct face wash technique such as being gentle is also important to obtain the best result. ${ }^{14}$

In this research, the majority of respondents used moisturizers. However, there was no relation between usage of moisturizers and type of moisturizers with the degree of acne 
vulgaris. This result is in line with the study by Rahmawati et al. ${ }^{14}$, which also shows no significant relation between the use of moisturizers and acne vulgaris $(p=0.520)$. The absence of a significant association could be due to the type of moisturizer used did not contain oil and comedogenic products. Frequently, moisturizers were suggested by some clinician to patients as adjuvant care of acne when benzoyl peroxide or retinoid was used in the topical treatment.

A study shows when moisturizer is used with adapalene (topical steroids), there is no effect on its therapeutic effect. Despite that, it helps to improve adherence to therapy with adapalene by decreasing the risk of experiencing uncomfortable skin symptoms such as dry skin conditions. ${ }^{15}$ At present, numerous moisturizers claim to be appropriate for acne care by functioning independently in preventing worsening of acne. Furthermore, $92 \%$ out of 52 moisturizers had antiinflammatory properties apart from occlusive, humectant and emollient effect when its active ingredients and properties were examined. Moisturizers that have anti-inflammatory properties contain anti-acne medication such as salicylic acid (35\%), benzoyl peroxide $(10 \%)$ and retinol $(8 \%) .{ }^{16}$

In this study, there was no association between the usage of sunblock as facial protection and degree of acne vulgaris. Yet, according to theory, the presence of physical blockers $\left(\mathrm{ZnO}\right.$ and $\left.\mathrm{TiO}_{2}\right)$ in sunscreen could cause exacerbation of acne. Due to the its greasy property and large particle sizes, these physical blockers will block skin pores. Oily skin and acne skin are suggested to use spray or gel-based sunscreens. The contrast of the result from this study and theory could be due to the type of sunblock used were sunblock with microfine particles, which were recommended to be safe and effective for acne patients. The usage of sunblock is important as the chance of photosensitivity increases with the usage of topical retinoids (topical medication for acne patient). Thus, it is used to decrease the risk of sunburn. The absence of a significant association could also be due to the frequency of applying the facial protection. ${ }^{17} \mathrm{~A}$ further study regarding correlation between frequency of applying sunblock and degree of acne can be performed by another researcher.

The usage of exfoliators is not related with the degree of acne vulgaris in this study. According to theoretical expectation which states appropriate usage of exfoliators helps to get rid of excess skin cells and clogging up acne pores. This is because exfoliation fastens skin cell regeneration and reduces comedones. This could be the reason of the absence of the significant association. However, Kubba et $\mathrm{al}^{4}{ }^{4}$ states that excessive usage of exfoliators especially on acne lesions will cause trauma and worsen the condition. A study regarding the frequency of using exfoliators and the degree of acne vulgaris or occurrence of acne vulgaris should be done for a better understanding of the relation of exfoliator and acne vulgaris. ${ }^{4}$

In this study, most of the respondents tended to avoid the frequent usage of decorative cosmetics as they only used decorative cosmetics on special occasions. This could be due to the common belief that decorative cosmetics are occlusive and can lead to cosmetic acne.$^{18}$ However, there is no association between the frequency of applying decorative cosmetics and degree of acne vulgaris. A case control study also suggests that overall cosmetic use is negatively linked with post-adolescent acne, but the use of some individual cosmetics have a positive effect with it, not all cosmetics causes acne. ${ }^{19}$

No significant association between the type of decorative cosmetics and degree of acne vulgaris was found in this study too. However, the study showed make up foundation was the most frequently used decorative cosmetics. It could be used to cover flaws such as acne scar. Usages of oil-free concealers, noncomedogenic makeup were reported to enhance the quality of life of individuals. Some foundations can also function as a moisturizer and sunscreen. ${ }^{3}$

Many other factors were found to be linked with the degree of acne vulgaris including skin type, skin sensitivity, hormonal, genetic, stress, infection and trauma. These factors were known to cause acne exacerbation. Yet, none of these factors were significantly associated with the degree of acne vulgaris in this study.

The number of populations in this study was small; this could be one of the factors that caused most of the variable to have a different result compared with other studies. The population of patients with moderate acne and severe acne was also limited in this study, as the majority of patients at the Outpatient clinic were patients with mild acne. Hence, a further investigation should increase the total sample size in order to overcome this problem. Due to the nature of this study, the cause and effect of diet and cosmetic on acne vulgaris could not be determined. A further research should use a better study design such as casecontrol, cohort etc. Also, the diet tracking was 
limited to a few types of foods. Therefore, further research should include a long-term diet tracking using food diary to have a better understanding on the effect of diets.

In this study, it is concluded that white rice, chocolate and oily food are associated with the degree of acne vulgaris. However, diet such as white rice, potatoes and milk have no influence on the degree of acne vulgaris. Moreover, cosmetics including moisturizer, cleansers, exfoliators, facial protection and decorative cosmetics are also not associated with the degree of acne vulgaris. Other than topical and oral medication for acne vulgaris, physicians should explore the factors that cause acne on different individuals to provide a more suitable treatment plan. Physicians should also educate patients the importance of avoiding certain diet and usage of cosmetic products that will cause an aggravation to the condition to speed up the healing process of the acne condition.

\section{References}

1. Goldsmith LI, Gilchrest BA, Paller AS, Leffell DJ, Wolff K. Fitzpatrick's dermatology in general medicine. $8^{\text {th }} \mathrm{Ed}$. New York: McGraw-Hill; 2012.

2. Sajayan J, Mohan A. Acne cosmeticacurrent burning issue. Int Ayurvedic Med J. 2015;3(4):1195-8.

3. Eichenfield LF, Krakowski AC, Piggott C, Del Rosso J, Baldwin H, Friedlander SF, et al. Evidence-based recommendations for the diagnosis and treatment of pediatric acne. Pediatrics. 2013;131 Suppl 3:S16386.

4. Kubba R, Bajaj A, Thappa D, Sharma R. Cosmetics and skin care in acne. Indian J Dermatology Venereol Leprol. 2009;75(7):S55-6.

5. Atkinson FS, Foster-Powell K, Brand-Miller JC. International tables of glycemic index and glycemic load values: 2008. Diabetes Care. 2008;31(12):2281-3.

6. Di Landro A, Cazzaniga S, Parazzini F, Ingordo V, Cusano F, Atzori L, et al. Family history, body mass index, selected dietary factors, menstrual history, and risk of moderate to severe acne in adolescents and young adults. J Am Acad Dermatol. 2012;67(6):1129-35.

7. Burris J, Rietkerk W, Woolf K. Relationships of self-reported dietary factors and perceived acne severity in a cohort of New York young adults. J Acad Nutr Diet.
Elsevier Inc; 2014;114(3):384-92.

8. Ismail NH, Abdul Manaf Z, Azizan NZ. High glycemic load, milk and ice cream consumption are related to acne vulgaris in Malaysian young adults: a case control study. BMC Dermatol. 2012;12(1):13.

9. Ghodsi SZ, Orawa H, Zouboulis CC. Prevalence, severity, and severity risk factors of acne in high school pupils: a community-based study. J Invest Dermatol. 2009;129(9):2136-41.

10. Reynolds RC, Lee S, Choi JYJ, Atkinson FS, Stockmann KS, Petocz P, et al. Effect of the glycemic index of carbohydrates on acne vulgaris. Nutrients. 2010;2(10):1060-72.

11. Caperton C, Block S, Viera M, Keri J, Berman B. Double-blind, placebo-controlled study assessing the effect of chocolate consumption in subjects with a history of acne vulgaris. J Clin Aesthet Dermatol. 2014;7(5):19-23.

12. Adebamowo CA, Spiegelman D, Berkey CS, Danby FW, RockettHH,Colditz GA, etal. Milk consumption and acne in teenaged boys. J Am Acad Dermatol. 2008;58(5):787-93.

13. Çerman AA, Aktaş E, Altunay IK, Arici JE, Tulunay A, Ozturk FY. Dietary glycemic factors, insulin resistance, and adiponectin levels in acne vulgaris. J Am Acad Dermatol. 2016;75(1):155-62.

14. Rahmawati D, Widayati RI, Sudaryanto S. Hubungan Perawatan Kulit Wajah dengan Timbulnya Akne Vulgaris pada Siswi SMA/ MA/SMK yang Menderita Akne Vulgaris. Jurnal Media Medika Muda. 2012;1(1):117.

15. Hayashi N, Kawashima M. Study of the usefulness of moisturizers on adherence of acne patients treated with adapalene. J Dermatol. 2014;41(7):1-6.

16. Chularojanamontri L, Tuchinda P, Kulthanan K, Pongparit K. Moisturizers for acne: What are their constituents? J Clin Aesthet Dermatol. 2014;7(5):36-44.

17. Latha MS, Martis J, Shobha V, Shinde RS, Bangera S, Krishnankutty B, et al. Sunscreening agents: A review. J Clin Aesthet Dermatol. 2013;6(1):16-26.

18. Tahir CM, Ansari R. Beliefs, perceptions and expectations among acne patients. J Pakistan Assoc Dermatologists. 2012;22:98-104.

19. Singh S, Mann BK, Tiwary NK. Acne cosmetica revisited: A case-control study shows a dose-dependent inverse association between overall cosmetic use and post-adolescent acne. Dermatology. 2013;226(4):337-41. 